\title{
Reconstruction based Finger-Knuckle-Print Verification with Score Level Adaptive Binary Fusion
}

\author{
Guangwei Gao ${ }^{\mathrm{a}, \mathrm{b}}$, Lei Zhang ${ }^{\mathrm{b}, 1}$, Jian Yang ${ }^{\mathrm{a}}$, Lin Zhang ${ }^{\mathrm{c}}$, David Zhang ${ }^{\mathrm{b}}$ \\ ${ }^{a}$ School of Computer Science and Engineering, Nanjing University of Science and Technology, Nanjing, \\ China \\ ${ }^{b}$ Department of Computing, The Hong Kong Polytechnic University, Hong Kong \\ ${ }^{\mathrm{c}}$ School of Software Engineering, Tongji University, Shanghai, China
}

\begin{abstract}
Recently a new biometrics identifier, namely finger knuckle print (FKP), has been proposed for personal authentication with very interesting results. One of the advantages of FKP verification lies in its user friendliness in data collection. However, the user flexibility in positioning fingers also leads to certain degree of pose variations in the collected query FKP images. The widely used Gabor filtering based Competitive Coding scheme is sensitive to such variations, resulting in many false rejections. We propose to alleviate this problem by reconstructing the query sample with a dictionary learned from the template samples in the gallery set. The reconstructed FKP image can reduce much the enlarged matching distance caused by finger pose variations; however, both the intra-class and inter-class distances will be reduced. We then propose a score level adaptive binary fusion rule to adaptively fuse the matching distances before and after reconstruction, aiming to reduce the false rejections without increasing much the false acceptances. Experimental results on the benchmark PolyU FKP database show that the proposed method significantly improves the FKP verification accuracy.
\end{abstract}

Keywords: Biometrics, finger-knuckle-print, reconstruction, score level fusion

\footnotetext{
${ }^{1}$ This work is supported by the HK RGC PPR grant (PolyU5019-PPR-11), the National Science Fund for Distinguished Young Scholars under grants no. 61125305 and no. 61233011, and the Key Project of Chinese Ministry of Education under grant no. 313030 .
} 


\section{Introduction}

Reliable personal identity verification is crucial in a variety of applications such as building access control, e-banking, airport, computer system login, and mobile phones, etc. Compared with the passwords or ID cards based solutions, biometrics authentication is much more preferable and reliable to those applications requiring high security. In the past decades, various biometrics traits, such as face [1, 2], fingerprint $[3,4]$, iris $[5,6]$, etc., have been widely studied. Meanwhile, hand-based biometrics methods are popular in the biometrics community, and techniques such as palmprint [7-9], hand geometry [10], hand vein [11], etc., have been developed. Nonetheless, every biometrics identifier has its pros and cons, and no biometric identifier can supersede the other one. Therefore, it is desired that new biometrics identifiers, as well as the associated feature extraction and pattern recognition techniques, can be further developed to improve and enrich the biometrics methods and technologies.
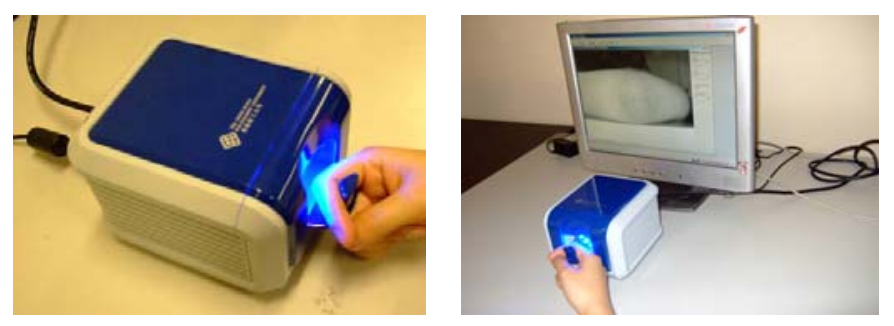

Figure 1: Outlook of the FKP image acquisition device.

Recently, a novel biometric identifier, namely finger-knuckle-print (FKP), has been developed for online personal verification [12-16]. FKP refers to the inherent skin pattern of the outer surface around the phalangeal joint of one's finger. Fig. 1 shows the FKP image acquisition device and the use of the system. Preprocessing and region of interest (ROI) extraction algorithms for FKP images can be found in $[12,13]$. In [12], Zhang et al. applied the Gabor filtering based Competitive Coding (CompCode) scheme, which was originally proposed for palmprint recognition [8], to the FKP image for orientation feature extraction and matching. In [13], this scheme was extended by combining the Gabor filtering magnitude information. The Gabor filtering based methods extract image local features. In [14], the Fourier transform based band-limited phase only correlation (BLPOC) was adopted to extract the global features of FKP images for matching, and in [15] the local and global information was combined for FKP verification, which achieves very promising 
accuracy of FKP verification. Recently, a set of phase congruency induced local features were defined in [16]. By fusing those local features in the matching score level, the proposed local feature integration (LFI) method [16] leads much better results than other local feature based methods such as CompCode [12] and improved CompCode [13].

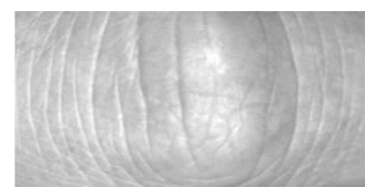

(a)
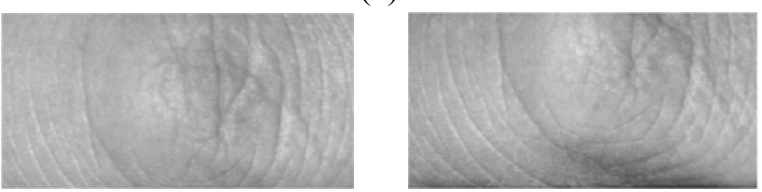

(b)

Figure 2: (a) A template FKP sample and (b) two testing FKP samples from the same finger but collected at different sessions. Obvious pose variations can be observed from the samples in (b).

Refer to Fig. 1, although a triangular block is used to control the finger freedom in FKP image acquisition, there is still much flexibility for the users to position their fingers. This is good to increase the user friendliness but also allows much variation of the finger pose in query sample collection process. Fig. 2 shows some examples. We can see obvious deformations between the two FKP samples due to the finger pose variations. Unfortunately, the CompCode and LFI based FKP recognition methods are sensitive to such variations, resulting in false rejections and degrading the FKP verification performance.

From the above discussions, we can see that the main difficulty in FKP recognition is the false rejections caused by finger-pose-variation in the query samples. One strategy to solve this problem is to correct the pose deformations by affine transformations. However, estimating the affine transformation parameters is itself a very difficult problem, particularly for FKP images where very few distinctive key points can be extracted. Since our ultimate goal is FKP verification but not pose deformation correction, another strategy is to enhance the FKP matching process without pose deformation correction. Considering that the finger pose variation caused FKP image deformation enlarges the matching distance between two FKP images from the same person and hence results in false rejections, we propose a reconstruction based matching scheme to reduce the enlarged matching distance. 
First, a dictionary is learned from the template FKP images, and this dictionary defines the subspace of the gallery FKP dataset. For a given query sample which may have pose variation, we represent it as the linear combination of the atoms in the learned dictionary. This process actually projects the query sample onto the subspace spanned by the gallery FKP images. The CompCode scheme can then be applied to the reconstructed image for feature extraction and matching. Nonetheless, the reconstruction of the query sample will not only reduce the intra-class distance, but also reduce the inter-class distance. In other words, it can reduce false rejections but may also increase the false acceptances. To effectively exploit the discriminative information of the query sample before and after reconstruction, a simple yet powerful score level adaptive binary fusion (ABF) rule is proposed to make the final decision by fusing the matching scores before and after reconstruction. The ABF ensures a good reduction of false rejections without increasing much the false acceptances, leading to much lower equal error rates than state-of-the-art methods.

The rest of this paper is organized as follows. Section 2 briefly reviews the CompCode scheme and indicates its problems. Section 3 presents the proposed reconstruction based methodology for FKP recognition. Section 4 presents the ABF rule. Section 5 summaries the proposed algorithm. Section 6 presents extensive experimental results and discussions, and Section 7 concludes the paper.

\section{Brief Review of Competitive Coding based FKP Verification}

Gabor filtering has been widely used as an effective feature extraction technique in face, iris, fingerprint, palmprint, as well as FKP recognition systems. A 2D Gabor filter can be mathematically expressed as

$$
G(n, m)=\exp \left(-\frac{1}{2}\left(\frac{n_{\theta}^{2}}{\sigma_{n}^{2}}+\frac{m_{\theta}^{2}}{\sigma_{m}^{2}}\right)\right) \cdot \exp \left(i 2 \pi f n_{\theta}\right)
$$

where $n_{\theta}=n \cdot \cos \theta+m \cdot \sin \theta, m_{\theta}=-n \cdot \sin \theta+m \cdot \cos \theta, f$ is the frequency of the sinusoid factor, $\theta$ is the orientation of the normal to the parallel stripes of the Gabor function, $\sigma_{n}$ and $\sigma_{m}$ are the standard deviations of the 2D Gaussian envelop.

Based on the observation that the FKP images contain abundant line-like structures, the orientation features can be extracted for FKP image recognition. Let's denote by $G_{R}$ the real part of a Gabor filter, and by $I_{R O I}$ an FKP ROI (region of interest) image. With a bank of Gabor filters, at each pixel $I_{R O I}(n, m)$, the CompCode scheme $[8,12,13]$ extracts and codes the dominant orientation feature as follows: 


$$
\operatorname{CompCode}(n, m)=\arg \min _{j}\left\{I_{R O I}(n, m) * G_{R}\left(n, m, \theta_{j}\right)\right\}
$$

where symbol "**" denotes the convolution operation, and $\theta_{j}=j \pi / 6, j=\{0, \ldots, 5\}$. CompCode $(n, m)$ is assigned the orientation along which the smallest response is obtained.

In order for real-time recognition, CompCode uses three bits to represent each orientation [8]. For matching two CompCode maps $P$ and $Q$, the normalized Hamming distance based angular distance is commonly adopted [8]:

$$
s_{h}=\frac{\sum_{n=1}^{\text {Rows }} \sum_{m=1}^{\text {Cols }} \sum_{i=0}^{2}\left(P_{i}(n, m) \otimes Q_{i}(n, m)\right)}{3 S}
$$

where $S$ is the area of the code map, $P_{i}\left(Q_{i}\right)$ is the $i^{\text {th }}$ bit plane of $P(Q)$, and $\otimes$ represent the bitwise "exclusive OR" operation. In practice, multiple matches are performed by translating one of the two feature maps vertically and horizontally, and the minimum matching distance is regarded as the final angular distance. Nonetheless, the CompCode scheme is sensitive to the variation of FKP image and then resulting in false rejection. Even a small rotation and misalignment can lead to an incorrect matching.

\section{Recognition via Reconstruction}

\subsection{Motivation}

The CompCode scheme is simple and fast, and it leads to acceptable accuracy in FKP verification [12, 13]. If the query FKP image is well aligned after ROI extraction, the CompCode scheme can work very well. As we discussed in the introduction section, however, there can be certain degree of variations of the finger pose in the data collection process (refer to Fig. 2 please), which lead to deformations in the FKP images and consequently result in false rejections because CompCode is sensitive to image deformations. 


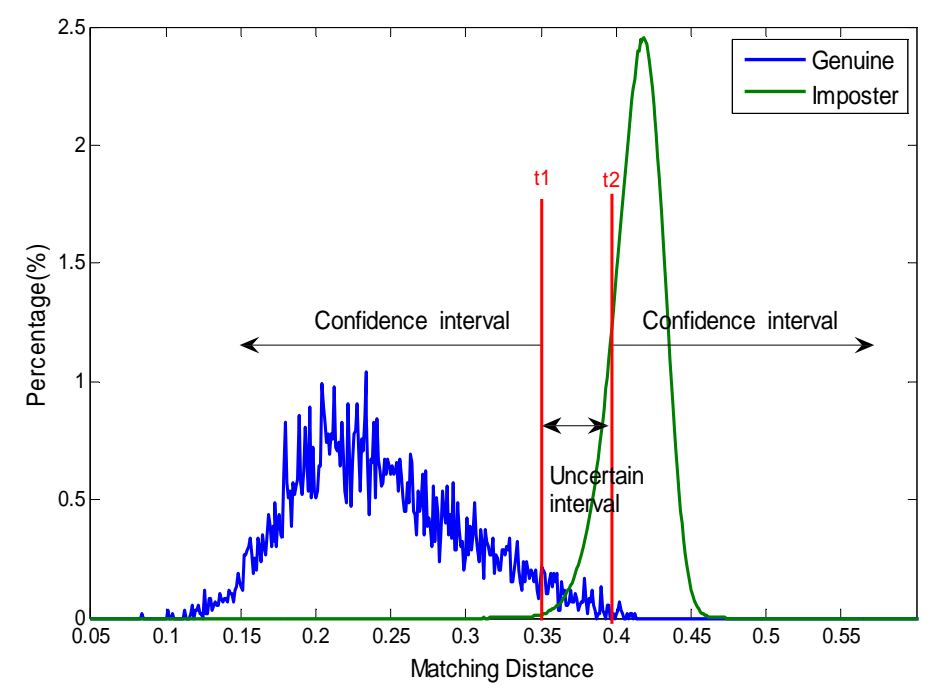

Figure 3: The distribution of matching distance of a typical FKP verification system.

Fig. 3 shows the genuine and imposter matching distance distributions of a typical FKP verification system. Based on the curves, it is obvious that we can divide the matching distance into two parts: an uncertain interval, which is between thresholds $t_{1}$ and $t_{2}$, and a confidence interval, which covers the remaining part. Generally speaking, if the matching distance of two FKP feature maps falls into the confidence interval, it can be easily decided if the query sample is a genuine or an imposter, while most of the false acceptances and false rejections occur when the matching distance falls into the uncertain interval $\left[t_{1}, t_{2}\right]$. On the other hand, the finger pose variation is the main cause that increases the intra-class distance (see the long tail of the blue genuine curve in Fig. 3), making the false rejections happen.

The FKP verification accuracy can be improved if we could correct the finger-pose-variation caused deformations of query samples via affine transformation. Unfortunately, it is a particularly difficult problem to estimate the affine transformation parameters for FKP images because very few distinctive key points can be extracted from them. Therefore, this solution is impractical. In this paper, we propose to reduce the pose variation caused false rejections by enhancing the matching process without pose deformation correction.

Denote by $\boldsymbol{X}=\left[\boldsymbol{x}_{1}, \boldsymbol{x}_{2}, \ldots, \boldsymbol{x}_{k}\right]$ the set of gallery FKP samples, where $\boldsymbol{x}_{i}$ is a vectorized FKP sample. We can use the hull of $\boldsymbol{X}$, denoted by $H(\boldsymbol{X})=\{\boldsymbol{X} \cdot \boldsymbol{w}\}$, where $\boldsymbol{w}=\left[w_{1}, \ldots, w_{k}\right]^{T}$ is the vector of weights, to characterize the subspace of gallery FKP images. If the gallery set is big enough, we can reasonably assume that each regular FKP image will fall into the hull $\boldsymbol{H}(\boldsymbol{X})$, i.e., it can be well represented as the linear 
combination of the template samples in $\boldsymbol{X}$. For a query image $\boldsymbol{y}$, we can project it into the hull $\boldsymbol{H}(\boldsymbol{X})$, and rewrite it as $\boldsymbol{y}=\boldsymbol{X} \cdot \hat{\boldsymbol{w}}+\boldsymbol{e}$, where weight $\hat{\boldsymbol{w}}$ is determined by minimizing the distance between $\boldsymbol{y}$ and its projection $\hat{\boldsymbol{y}}=\boldsymbol{X} \cdot \hat{\boldsymbol{w}}$ (usually some regularization will be imposed on $\hat{\boldsymbol{w}}$ ), and $\boldsymbol{e}$ is the projection residual.

For a regular query image, there is no much finger pose variation caused deformation in it, and the projection residual $\boldsymbol{e}$ will be very small (i.e., $\|\boldsymbol{e}\|_{2}$ is very small). In such case, the query sample $\boldsymbol{y}$ can be accurately recognized by using the efficient CompCode scheme. However, if the finger pose varies much in the data acquisition process, the query sample $\boldsymbol{y}$ can have much deformation and fall outside the hull $H(\boldsymbol{X})$, i.e., $\|\boldsymbol{e}\|_{2}$ becomes much bigger. In this case, the CompCode scheme can fail since it is sensitive to image deformations, which can be reflected by the big values of $\|\boldsymbol{e}\|_{2}$.

Let's plot the distribution of $\|\boldsymbol{e}\|_{2}$ by using the PolyU FKP database [32]. We use the first 6 samples of each of the 660 classes in the PolyU FKP database to construct the gallery dataset $\boldsymbol{X}$, and take the remaining 6 samples of each class as the query samples. In projecting each query sample into the hull $H(\boldsymbol{X})$, we use the $l_{2}$-norm regularized least square to compute the weight: $\hat{\boldsymbol{w}}=\arg \min _{\boldsymbol{w}}\|\boldsymbol{y}-\boldsymbol{X} \cdot \boldsymbol{w}\|_{2}^{2}+\lambda\|\boldsymbol{w}\|_{2}^{2}$. Then $\boldsymbol{e}$ is computed as $\boldsymbol{e}=\boldsymbol{y}-\boldsymbol{X} \cdot \hat{\boldsymbol{w}}$. In Fig. 4 we plot the distribution of $\|\boldsymbol{e}\|_{2}$ by using all the $660 \times 6=3,960$ query samples. We can see that the distribution has a long tail, which is mainly caused by those samples with large deformations. Since it is mainly the big residual $\boldsymbol{e}$ that makes the CompCode feature of query sample $\boldsymbol{y}$ deviates much from the CompCode features of gallery samples in $\boldsymbol{X}$, one intuitive idea is that we can compute the CompCode of $\hat{\boldsymbol{y}}=\boldsymbol{X} \cdot \hat{\boldsymbol{w}}$ for verification.

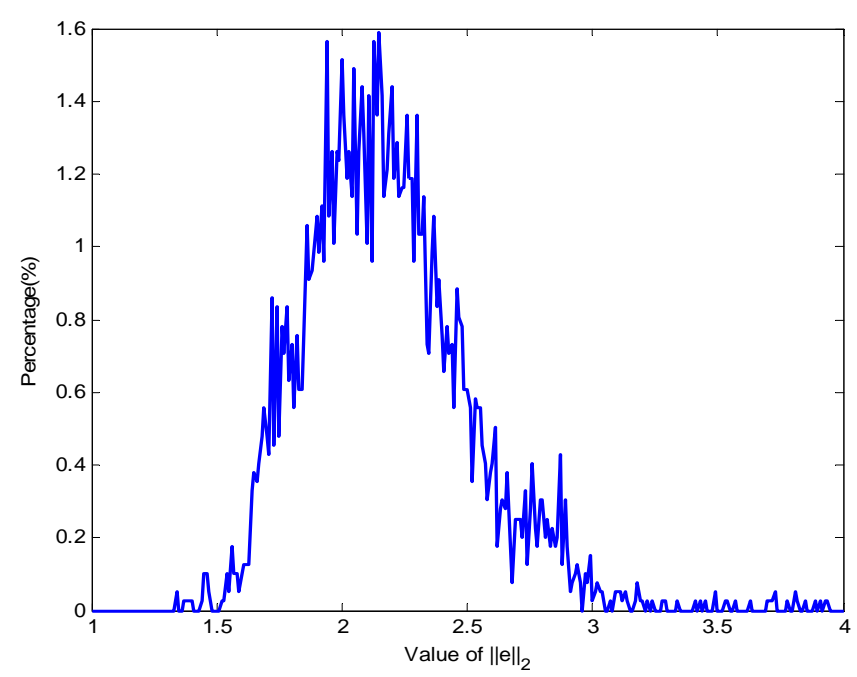

Figure 4: The distribution of $\|\boldsymbol{e}\|_{2}$. 
Note that we use all the gallery samples, not only the samples from the class that $y$ claims, to compute $\hat{\boldsymbol{y}}$. There are two reasons for such a configuration. First, the number of samples of each class is usually small (e.g., 6 samples per class in the gallery set of PolyU FKP database), and thus reconstructing $y$ using only the samples from one class is not accurate. Second, the query sample $y$ can be an imposter, i.e., it may come from a class out of the gallery set, or from a class that is different from the class it claims. Reconstructing $y$ using only the samples of one class will make the distance from $\hat{\boldsymbol{y}}$ to this class too small so that false acceptance will happen. Therefore, all the classes in the gallery set should be involved in reconstructing $y$.

\subsection{Reconstruction with $l_{1}$-norm sparse regularization}

By approximating $\boldsymbol{y}$ with $\boldsymbol{X} \cdot \boldsymbol{w}$, one solution to $\boldsymbol{w}$ is the least square solution: $\hat{\boldsymbol{w}}=\arg \min _{\boldsymbol{w}}\|\boldsymbol{y}-\boldsymbol{X} \cdot \boldsymbol{w}\|_{2}^{2}$. It is easy to see that $\hat{\boldsymbol{w}}=\left(\boldsymbol{X}^{T} \boldsymbol{X}\right)^{-1} \boldsymbol{X}^{T} \boldsymbol{y}$. Though the least square solution is simple to compute and it ensures the minimal $l_{2}$-norm reconstruction residual of $\hat{\boldsymbol{y}}$, it is not the best choice for the verification purpose. The least square solution aims to minimize the reconstruction residual, and the weights $w$ tend to be densely distributed, hence many classes in $\boldsymbol{X}$ will contribute in reconstructing $\boldsymbol{y}$. Finally, some discriminative features in $\boldsymbol{y}$ may be smoothed out in $\hat{\boldsymbol{y}}$.

In order to preserve the discriminative features of $\boldsymbol{y}$ in $\hat{\boldsymbol{y}}$, some regularization term could be imposed on $w$. Intuitively, we hope that only a small portion of the weights in $w$ are significant so that only several classes are dominantly involved to reconstruct $y$. The $l_{1}$-norm based sparse representation (or sparse coding) is a very good choice to this end. In recent years sparse coding has been successfully used in various image reconstruction and pattern classification applications [17-22]. It represents a given signal as a sparse linear combination over a dictionary of atoms. By imposing the $l_{1}$-norm constraint on $\boldsymbol{w}$, we have

$$
\hat{\boldsymbol{w}}=\arg \min _{\boldsymbol{w}}\|\boldsymbol{y}-\boldsymbol{X} \cdot \boldsymbol{w}\|_{2}^{2}+\lambda\|\boldsymbol{w}\|_{1}
$$

where $\lambda$ is a positive scalar balancing the reconstruction residual and the sparsity of $\boldsymbol{w}$. Eq. (4) can be solved by many convex optimization algorithms such as $l_{1}$-magic [17], $l_{1}-l s[18]$, etc.

The sparse coding in Eq. (4) still has two problems. First, it is known that the commonly used $l_{1}$-minimization solvers such as $l_{1} l s$ have an empirical complexity of $O\left(z^{2} k^{1.3}\right)$ [18], where $z$ is the dimension of $\boldsymbol{y}$ and $k$ is the number of samples in $\boldsymbol{X}$. In practice, $k$ can be very big so that the sparse coding complexity 
is high. Second, the atoms in $\boldsymbol{X}$ are the original gallery FKP images, which may contain noise and some trivial structures that can be negative to the representation of $y$.

To solve the above problems and considering the fact that the FKP images in $\boldsymbol{X}$ have much redundancy across samples, we can learn a more compact dictionary $\boldsymbol{D}$ from $\boldsymbol{X}$, and then use $\boldsymbol{D}$ to code the input FKP image $y$. Dictionary learning has been widely used in image processing and pattern recognition [19-21, 23, 24]. In this paper, we simply adopt the method in [21] to learn the dictionary $\boldsymbol{D}$. Denote by $\boldsymbol{D}=\left[\boldsymbol{d}_{1}, \boldsymbol{d}_{2}, \ldots, \boldsymbol{d}_{p}\right]$, where $p \leq k$ and each $\boldsymbol{d}_{j}$ is a unit column vector. The dictionary learning can be formulated as the following minimization problem:

$$
J_{D, W}=\arg \min _{\boldsymbol{D}, \boldsymbol{W}}\left\{\|\boldsymbol{X}-\boldsymbol{D W}\|_{F}^{2}+\lambda\|\boldsymbol{W}\|_{1}\right\}
$$

Eq. (5) is a joint optimization of dictionary $\boldsymbol{D}$ and the coefficient matrix $\boldsymbol{W}$, and it can be solved by optimizing $\boldsymbol{D}$ and $\boldsymbol{W}$ alternatively [21]. Once the dictionary $\boldsymbol{D}$, which has less number of atoms than $\boldsymbol{X}$, is computed, we use it to code the input FKP image $y$ as follows:

$$
\hat{\boldsymbol{w}}=\arg \min _{\boldsymbol{w}}\|\boldsymbol{y}-\boldsymbol{D} \cdot \boldsymbol{w}\|_{2}^{2}+\lambda\|\boldsymbol{w}\|_{1}
$$

Finally, the image is reconstructed as:

$$
\hat{\boldsymbol{y}}=\boldsymbol{D} \cdot \hat{\boldsymbol{w}}
$$
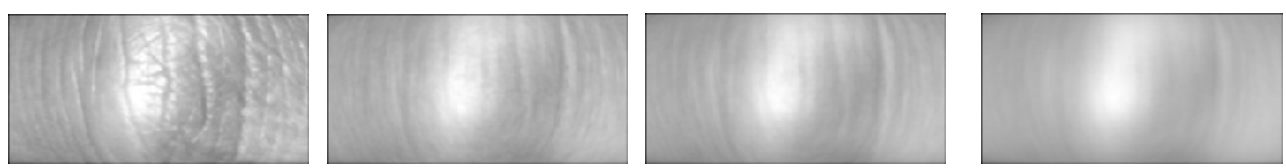

Figure 5: From left column to right column: original FKP image; reconstructed FKP image by $l_{1}$-regularization (Eq. (6)), reconstructed FKP image by $l_{2}$-regularization (Eq. (8)), and reconstructed FKP image without regularization (i.e., let $\lambda=0$ ).

Let's show an example of the reconstruction results. We take 1980 images from the first 330 classes in the gallery set of PolyU FKP dataset as $\boldsymbol{X}$, and learn from it a dictionary $\boldsymbol{D}$ with 1386 atoms. $\boldsymbol{D}$ is then used to reconstruct the input image $y$. The parameter $\lambda$ is set as 0.1 and 2 in Eq. (5) and Eq. (6), respectively. The left column of Fig. 5 shows an input FKP image, and the second column shows the reconstructed image using $\boldsymbol{D}$. One can see that the reconstructed image is smoother than the original one because some details as well as variations in the query sample $\boldsymbol{y}$, which cannot be represented by the learned dictionary $\boldsymbol{D}$, are suppressed. 
In Eq. (6), the $l_{1}$-norm sparsity constraint is imposed on the coding coefficients to enforce that only a small portion of the atoms are dominantly used to reconstruct $y$. However, $l_{1}$-minimization is time consuming. Though many fast $l_{1}$-minimization solvers such as FISTA [25], ALM [26] and Homotopy [27] have been developed, they may not be fast and accurate enough for practical use in the application of FKP verification, where real time implementation is expected. One intuitive solution is to relax the strong $l_{1}$-regularization to the weaker $l_{2}$-regularization in Eq. (6). The $l_{2}$-regularization offers a closed form solution to $w$, which can be very efficiently computed. Though the resolved coefficient $\boldsymbol{w}$ is not sparse anymore, the $l_{2}$-regularization can still make $w$ have a small energy. As we will see in the experimental results, the FKP verification accuracy by $l_{2}$-regularized reconstruction is only a little lower than that by $l_{1}$-regularized reconstruction, but the computational complexity is greatly reduced.

By using $l_{2}$-norm to regularize $\boldsymbol{w}$, the coding becomes a regularized least square problem:

$$
\hat{\boldsymbol{w}}=\arg \min _{w}\|\boldsymbol{y}-\boldsymbol{D} \cdot \boldsymbol{w}\|_{2}^{2}+\lambda\|\boldsymbol{w}\|_{2}^{2}
$$

and a closed form solution can be readily obtained as:

$$
\hat{\boldsymbol{w}}=\left(\boldsymbol{D}^{T} \boldsymbol{D}+\lambda \cdot \boldsymbol{I}\right)^{-1} \boldsymbol{D}^{T} \boldsymbol{y}
$$

Let $\boldsymbol{P}=\left(\boldsymbol{D}^{T} \boldsymbol{D}+\lambda \cdot \boldsymbol{I}\right)^{-1} \boldsymbol{D}^{T}$. Clearly, $\boldsymbol{P}$ can be pre-calculated so that the coding vector can be obtained by projecting $\boldsymbol{y}$ over $\boldsymbol{P}: \hat{\boldsymbol{w}}=\boldsymbol{P} \boldsymbol{y}$. This process is very fast.

In the third column of Fig. 5, we show the reconstructed FKP image by $l_{2}$-regularization in Eq. (8). The parameter $\lambda$ is set as 4 . (Note that the parameter $\lambda$ in $l_{2}$-regularized coding is usually set bigger than that in $l_{1}$-regularized coding because $l_{2}$-regularization is much weaker than $l_{1}$-regularization.) For a better illustration, we also show in the right column of Fig. 5 the reconstructed FKP image without regularization (i.e., set $\lambda=0$ in Eq. (8)). It can be observed that due to the non-sparse $l_{2}$-regularization, more classes are involved in the reconstruction of $\boldsymbol{y}$, and thus the reconstructed image is smoother than that by $l_{1}$-sparse regularization. This will lead to some lose of the distinctive features in the original FKP image. Nonetheless, this is the price we should pay for the great reduction in time complexity. In the section of experimental results, we will see that the reconstructed image by $l_{2}$-regularization can still lead to quite competitive verification accuracy. It can also be observed from Fig. 6 that the reconstructed image without regularization is the smoothest and more distinctive features are lost. Our experiments also show that the verification 
accuracy by the reconstructed image without regularization is lower than that with $l_{2}$-regularization ${ }^{2}$, which validates that $l_{2}$-regularization is very helpful for verification. Note that the complexity of reconstruction with $l_{2}$-regularization is the same as that without $l_{2}$-regularization.

\subsection{Patch based reconstruction}

In Sections 3.2 and 3.3, we stretch the whole FKP image as a vector $y$ for coding and reconstruction. In coding $\boldsymbol{y}$ over $\boldsymbol{D}$ by Eq. (6) or Eq. (8), we actually enforce that all the elements (i.e., all the pixels) in $\boldsymbol{y}$, denoted by $y_{i}, i=1,2, \ldots, n$, share the same coding vector $\boldsymbol{w}$ over their corresponding sub-dictionary (i.e., the $i^{\text {th }}$ row of $\boldsymbol{D}$ ). The good side of such a global coding strategy is that the solution is very stable because only one global coding vector needs to be solved. The bad side of such a coding strategy, however, lies in its less flexibility because it does not allow the different parts of the FKP image to have different coding vectors. Considering the fact that different portions of a query FKP sample $y$ may have different variations, it is reasonable to allow them to have different coding vectors so that the reconstruction can be spatially adaptive. Therefore, we can partition the FKP image into several patches, reconstruct separately each patch, and then combine them to obtain the whole reconstructed image.

If we partition the image into too many patches, the size of each patch will become small and the sub-dictionary corresponding to each patch will tend to be under-determined (i.e., the sub-dictionary will tend to be a fat matrix). This will reduce the stability of the coding process, no matter $l_{1}$-regularization or $l_{2}$-regularization is used. Based on our experimental experience and considering the special pattern of FKP images, we partition the FKP image (size: $110 \times 220$ ) into 6 overlapped patches, as illustrated in Fig. 6. Four patches of size $60 \times 45$ lie in the four corners of the FKP images, and two patches of size $60 \times 155$ lie in the middle of the image. The reason that we set two fat rectangle patches in the middle is based on the observation that the pose variation along vertical direction has bigger effect than that along horizontal direction on the FKP recognition accuracy. Therefore, we should pay more attention to the pose variation along vertical direction in the partition.

\footnotetext{
${ }^{2}$ For example, we use the first 165 classes in the first session as the gallery set and the whole 660 classes in the second session as the probe set. By using the algorithm described in Table 2 together with the ABF rule (refer to Section 4 please), if the query sample is reconstructed with $l_{2}$-regularization, the equal error rate is $1.27 \%$, while if the query sample is reconstructed without regularization, the equal error rate is $1.41 \%$. We see that the $l_{2}$-regularization is helpful for verification without increasing the time complexity.
} 


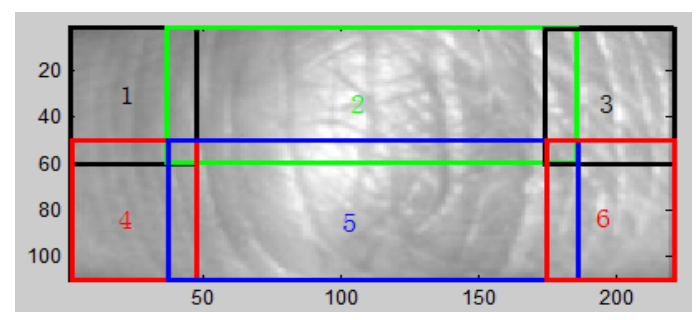

Figure 6: The patch partition of FKP images.

Let's denote by $\boldsymbol{y}_{j}, j=1,2, \ldots, 6$, the six patches of an FKP image $\boldsymbol{y}$. For each patch, we can learn a dictionary $\boldsymbol{D}_{j}$ from the training samples by using the same method described in Section 3.2. Then for an input query sample $\boldsymbol{y}$, each patch $\boldsymbol{y}_{j}$ of it can be reconstructed by $\boldsymbol{D}_{j}$. Similarly, both $l_{1}$-regularization and $l_{2}$-regularization can be employed in the reconstruction of $\boldsymbol{y}_{j}$ via

$$
\hat{\boldsymbol{w}}_{j}=\arg \min _{\boldsymbol{w}_{j}}\left\|\boldsymbol{y}_{j}-\boldsymbol{D}_{j} \cdot \boldsymbol{w}_{j}\right\|_{2}^{2}+\lambda\left\|\boldsymbol{w}_{j}\right\|_{1}
$$

and

$$
\hat{\boldsymbol{w}}_{j}=\arg \min _{\boldsymbol{w}_{j}}\left\|\boldsymbol{y}_{j}-\boldsymbol{D}_{j} \cdot \boldsymbol{w}_{j}\right\|_{2}^{2}+\lambda\left\|\boldsymbol{w}_{j}\right\|_{2}^{2}
$$

respectively. After each patch is reconstructed, the whole reconstructed image can be obtained by combining them. For the overlapped area of neighboring patches, we simply average the results. Similar to our discussions in Sections 3.2 and 3.3, the $l_{1}$-regularization in Eq. (10) may preserve more discrimination information, but the $l_{2}$-regularization in Eq. (11) is much faster.

At last, let's use an example to illustrate the performance of patched based reconstruction. Fig. 7 shows a query sample $\boldsymbol{y}$ and a template image which is from the same class of $\boldsymbol{y}$. We reconstruct $\boldsymbol{y}$ with $l_{2}$-regularization in this example. Image $\hat{\boldsymbol{y}}_{g}$ is the output of global reconstruction by Eq. (8) (we set $\lambda=1$ ), while $\hat{\boldsymbol{y}}_{p}$ is the output of patch based reconstruction by Eq. (11) (we set $\lambda=0.1$ ). One can see that the patch based reconstruction can preserve more distinctive features than the global reconstruction due to the higher flexibility in coding coefficients of each patch. After extract the CompCode feature maps (refer to Section 2 please), the matching distances between the template image and $\boldsymbol{y}, \hat{\boldsymbol{y}}_{g}$ and $\hat{\boldsymbol{y}}_{p}$ are $0.3783,0.3761$ and 0.3631, respectively. One can see that the global reconstruction can reduce the genuine matching distance, while the patched based reconstruction can further reduce the matching distance. Though imposter matching distance will also be reduced after reconstruction, we will see that with an adaptive binary fusion strategy proposed in next section, more accurate verification results can be obtained by adaptively fusing the 
matching distances before and after reconstruction. In the experimental results in Section 6, we will also see that patch based reconstruction can lead to better verification performance.

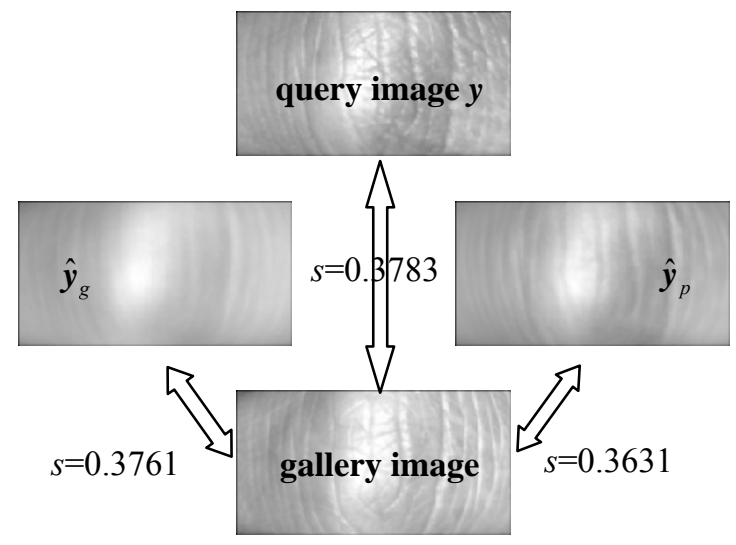

Figure 7: A query sample $y$ and the reconstructed images of it with $l_{2}$-regularization. $\hat{\boldsymbol{y}}_{g}$ is the output of global reconstruction while $\hat{\boldsymbol{y}}_{p}$ is the output of patch based reconstruction. The matching distances between a gallery image (which is from the same class as $\boldsymbol{y}$ ) and $\boldsymbol{y}, \hat{\boldsymbol{y}}_{g}$ and $\hat{\boldsymbol{y}}_{p}$ are $0.3783,0.3761$ and 0.3631 , respectively. One can see that the global reconstruction can reduce the genuine matching distance, while the patched based reconstruction can further reduce the matching distance.

\section{Verification by Binary Score Level Fusion}

As can be observed in Fig. 5 and Fig. 7, the reconstruction can reduce the deformation caused matching distance so that the intra-class matching distance can be reduced. At the same time, however, the inter-class matching distance may also be reduced. Fig. 8 shows some examples. One can see that the imposter inter-class matching distance is also reduced after the query sample is reconstructed (global reconstruction with $l_{2}$-regularization is used). What we expected is that the intra-class genuine matching distance can be reduced more than the inter-class imposter matching distance, but there is no guarantee for such an ideal situation. If we directly apply the CompCode scheme [8] to the reconstructed image for verification, incorrect decision can be made. To make this clear, we apply CompCode to the original images and the reconstructed images, respectively, for FKP verification. The equal error rates (EER) are shown in Table 1, from which we can see that using only the reconstructed images for verification leads to worse performance because some useful texture features are smoothed out in the reconstructed images. For a more robust and accurate verification, the matching scores (or distances) of both the original image $y$ and the reconstructed 


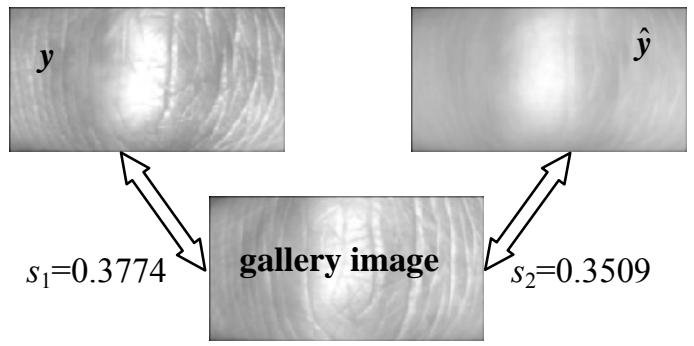

(a)

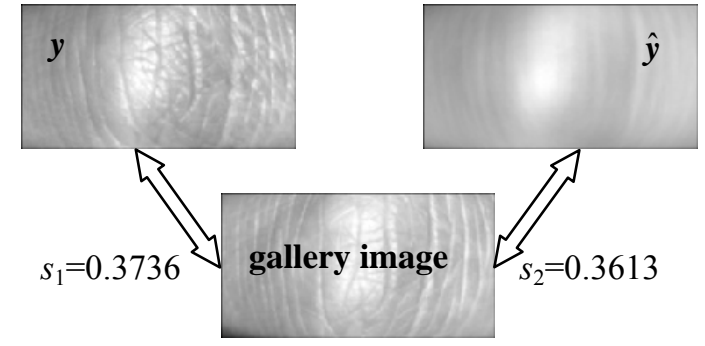

(b)

Figure 8: Examples of matching distances before and after reconstruction. (a) inter-class; (b) intra-class.

Table 1: FKP verification by using only the original image and only the reconstructed image, respectively.

\begin{tabular}{|c|c|c|}
\hline Images used & Original & Reconstructed \\
\hline EER & $1.65 \%$ & $2.09 \%$ \\
\hline
\end{tabular}

image $\hat{\boldsymbol{y}}$ should be considered for decision making.

Denote by $s_{1}$ and $s_{2}$ the matching distances of $\boldsymbol{y}$ and $\hat{\boldsymbol{y}}$ to a gallery image, respectively. We propose to fuse the two distance scores for final decision making. In the following, we first briefly review the existing popular score level fusion methods, and then propose a simple but very effective adaptive fusion method, namely the adaptive binary fusion rule.

\subsection{Popular score level fusion methods}

The score level fusion is a kind of combination-based approach, where the matching scores of individual matchers are integrated to generate a single scalar score for final decision making. Denote by $s$ the fusion result of $s_{1}$ and $s_{2}$. Three commonly used score level fusion methods [28] are the simple-sum (SS):

$$
s=s_{1}+s_{2}
$$

the min-score (MIN):

$$
s=\min \left\{s_{1}, s_{2}\right\}
$$

and the max-score (MAX):

$$
s=\max \left\{s_{1}, s_{2}\right\}
$$

The above three fusion rules do not use any additional information apart from the matching scores $s_{1}$ and $s_{2}$. Another popular method is the matcher weighting (MW) scheme [28]. The fused score is the weighted 
average of the two scores:

$$
s=\omega_{1} \cdot s_{1}+\omega_{2} \cdot s_{2}
$$

where $0 \leq \omega_{1}, \omega_{2} \leq 1$ and $\omega_{1}+\omega_{2}=1$. To determine $\omega_{1}$ and $\omega_{2}$, some prior knowledge needs to be known. Often the EERs of the two matchers (i.e., matching by $\boldsymbol{y}$ and matching by $\hat{\boldsymbol{y}}$ ) are used. Using some training dataset, the two EERs, denoted by $e_{1}$ and $e_{2}$, can be obtained, and the weight can then be calculated as

$$
\omega_{j}=\left(1 /\left(\sum_{j=1}^{2}\left(1 / e_{j}\right)\right)\right) / e_{j}
$$

It is obvious that the weight is inversely proportional to the corresponding EER. A higher weight will be assigned to a more reliable (i.e., lower EER) matcher, and vice versa.

There are also some other score level fusion formulas. For example, in [29] Kumar et al. proposed the exponential sum rule $s=\sum_{j=1}^{n} \exp \left(s_{j}\right) \omega_{j}$ and the tan-hyperbolic sum rule $s=\sum_{j=1}^{n} \tanh \left(s_{j}\right) \omega_{j}$. The Particle Swarm Optimization (PSO) [30] is employed to dynamically select the weights $\omega_{j}$. Such dynamic rules may work better than the SS, MIN, MAX and MW rules when there are multiple biometric identifiers for fusion, but they need to optimize the fusion rules, weights, and decision thresholds. Overall, the PSO based dynamic score level fusion is complex and has high computational complexity [29].

\subsection{Adaptive binary fusion}

In general, the MW fusion rule works better than SS, MIN and MAX rules. However, the MW rule has two drawbacks. First, it needs a preset training dataset to train the weights. Second, once the weights are learned, they are applied to all query images $\boldsymbol{y}$ and the reconstructed images $\hat{\boldsymbol{y}}$. In other words, the MW rule is not adaptive to the input query image, limiting its performance. This phenomenon can be seen in Fig. 8. In Fig. 8(b), the reconstructed image is more reliable for verification, so the weight $\omega_{2}$ assigned to this matcher is higher $\left(\omega_{1}<\omega_{2}\right)$. However, the situation is opposite in Fig. 8(a). Because the query sample is an imposter, it is hoped that the weight $\omega_{1}$ assigned to the original image is higher $\left(\omega_{1}>\omega_{2}\right)$ so that the final matching distance can be larger. The MW rule cannot meet this requirement because $\omega_{1}$ and $\omega_{2}$ are fixed.

In this paper, we propose a new fusion rule, which is adaptive to the query image and does not need a preset training dataset. The weights $\omega_{1}$ and $\omega_{2}$ are adaptively determined online based on the input image 
pair $\boldsymbol{y}$ and $\hat{\boldsymbol{y}}$. The idea is as follows. For the query image $\boldsymbol{y}$ which is claimed to belong to class $c$, we can calculate its within-class and between-class matching distances. Using CompCode, those matching distances can be computed very fast by Eq. (3). So does for the reconstructed query image $\hat{y}$. Then the higher weight is assigned to the matcher whose within-class and between-class matching distances are better separated.

Denote by $\mu_{1, w}$ and $\mu_{1, b}$ the mean values of the within-class and between-class matching distances of $\boldsymbol{y}$, respectively, and by $\sigma_{1, w}$ and $\sigma_{1, b}$ the standard deviations of the within-class and between-class matching distances, respectively. For both genuine matching and imposter matching, the within-class distances are calculated between the query image and the class which it claims to belong to, and the between-class distances are calculated between the query image and other classes. Because of the fast speed of CompCode, this process can be implemented in less than one second on the PolyU FKP database. For very large scale databases, computing the between-class distance using the whole dataset can be costly. To save cost, we can randomly select an enough number of samples from the classes other than the claimed class to compute a good approximation of the between-class distance.

The decidability index [31] can be used to measure the separability of the distributions of within-class and between-class matching distances. The decidability index for $\boldsymbol{y}$ is calculated as follows:

$$
d_{1}=\frac{\left|\mu_{1, w}-\mu_{1, b}\right|}{\sqrt{\left(\sigma_{1, w}^{2}+\sigma_{1, b}^{2}\right) / 2}}
$$

Similarly, we can calculate the decidability index of $\hat{y}$ and denote it as $d_{2}$. A bigger decidability index means that the within-class and between-class matching scores can be better separated, and hence the matcher is more accurate, vice versa. Therefore, $d_{1}$ and $d_{2}$ can be used to adaptively determine the weights $\omega_{1}$ and $\omega_{2}$ that are assigned to $s_{1}$ and $s_{2}$. The higher the decidability index, the higher the weight. However, designing an optimal function to map $d_{1}$ and $d_{2}$ to $\omega_{1}$ and $\omega_{2}$ is not a trivial work. In this paper, we propose to use the simple binary logic operation for the weight determination. The so-called adaptive binary fusion $(\mathrm{ABF})$ rule is defined as follows:

Adaptive Binary Fusion (ABF): If $d_{1} \geq d_{2}, \omega_{1}=1$ and $\omega_{2}=0$; otherwise, $\omega_{1}=0$ and $\omega_{2}=1$.

The above proposed ABF rule is a kind of "winner-take-all" strategy and is similar to the notion of using cohort scores for multi-biometric fusion [28]. However, there are clear differences between them. In cohort 
score based multi-biometric fusion, the fusion weight is fixed for each biometric identifier once the weights are learned offline. All the users of one biometric identifier share the same pre-learned fusion weight. Instead, in the proposed ABF the fusion weight is adaptively determined for each user online. The ABF rule is simple but fits our application very well. The reconstruction of $y$ can only lead to two situations: the reconstructed image $\hat{y}$ is either better or worse than $\boldsymbol{y}$ for verification. Hence, it is reasonable and effective to adaptively choose one of them for the final decision making. Our experimental results in Section 6 validate the effectiveness of the proposed $\mathrm{ABF}$ rule.

\section{Summary of the Verification Algorithm}

Table 2: Summary of the proposed FKP verification algorithm.

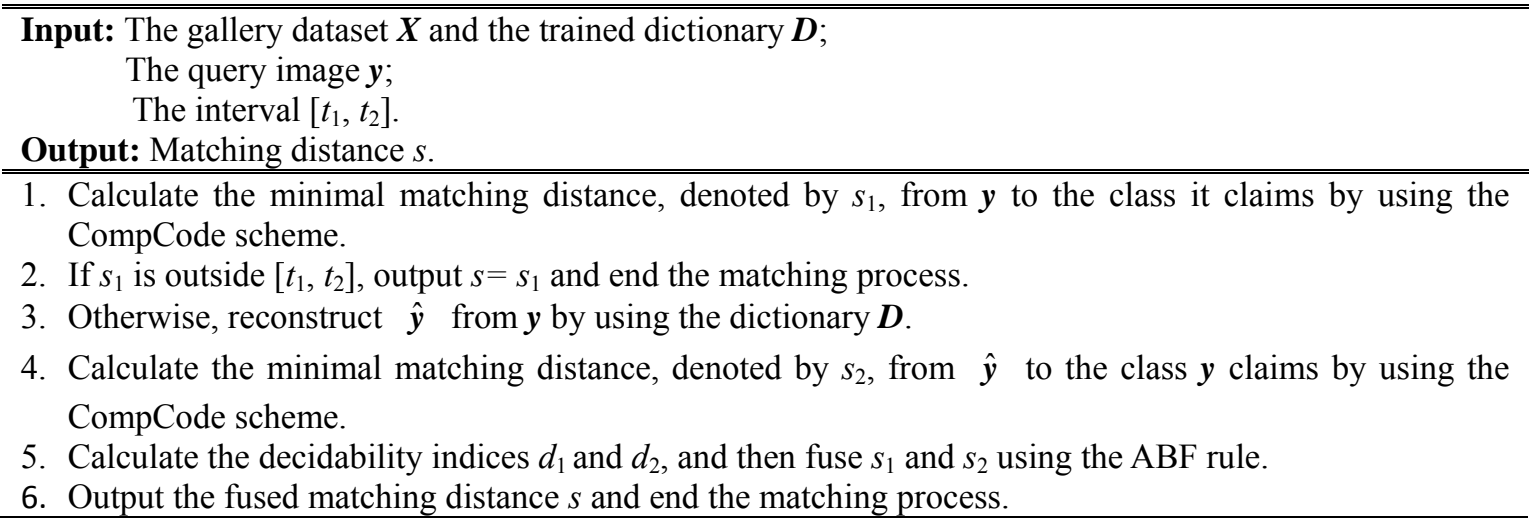

In practice, it is not necessary to reconstruct every input query image for verification. As shown in Fig. 4, if the matching distance of a query image $y$ falls into the confident interval, we can directly make the decision; only when the matching distance falls into the uncertain interval $\left[t_{1}, t_{2}\right]$, the reconstruction is needed, and the $\mathrm{ABF}$ rule is applied for the verification. The proposed algorithm of reconstruction based FKP verification with ABF is summarized in Table 2. The output is the final matching distance $s$. The final decision (accept or reject) is then made by applying a threshold to $s$, as in existing FKP verification systems [12-16].

\section{Experimental Results}


The PolyU FKP database, which can be freely downloaded at http://www.comp.polyu.edu.hk/ biometrics/ FKP.htm, was used in our experiments. This database contains the cropped FKP region of interest (ROI) images of 4 fingers (the left index finger, the left middle finger, the right index finger and the right middle finger) from 165 persons. Each finger knuckle was acquired 12 samples in two separated sessions with 6 samples per session, giving a total of $165 \times 4 \times 12=7,920$ samples from 660 (i.e., $165 \times 4$ ) fingers.

In the following experiments, the gallery set is always extracted from the first session while the probe set is extracted from the second session. As in [12-16], each image in the probe set was matched with all the images in the gallery set. If the two images were from the same finger, a genuine matching was counted; otherwise, an imposter matching was counted.

\subsection{Comparison between different fusion rules}

In this section, we verify that the proposed ABF rule is more effective than the commonly used SS, MIN, MAX and MW rules. In the experiment, the gallery set is composed of the first 165 fingers in the PolyU FKP gallery set, and the probe set is composed of the first 330 fingers in the PolyU FKP probe set. (Other settings of the gallery and probe sets lead to similar conclusions.) That is, there are 165 classes out of the gallery set. The gallery set is used to train the dictionary $\boldsymbol{D}$ to code the samples in the probe set.

Table 3: EER (\%) values by different fusion rules.

\begin{tabular}{|c|c|c|c|c|c|}
\hline Fusion rules & SS & MIN & MAX & MW & ABF \\
\hline EER & 2.22 & 2.07 & 2.33 & 1.99 & $\mathbf{1 . 1 0}$ \\
\hline
\end{tabular}

In this experiment the $l_{1}$-regularization is used in the reconstruction of query samples. In the dictionary learning (Eq. (5)) and sparse coding (Eq. (6)), the parameter $\lambda$ is set as 0.1 and 0.5 . The number of atoms in the learned dictionary is 0.7 times the number of samples in the gallery set. The parameters for uncertain interval setting is $t_{1}=0.35$ and $t_{2}=0.39$ based on our experimental experience. If the class label of the query sample is out of the gallery set, only the imposter matching distance will be counted when calculating EER.

The algorithm described in Table 2 is used to perform the FKP verification experiments with different fusion rules in step 5. For the MW rule, the whole dataset is used to train the weights. The EER results by using the different fusion rules are listed in Table 3. We can clearly see that the lowest EER is obtained by the proposed $\mathrm{ABF}$ rule, which works much better than other rules. Even that the MW rule uses more 
information with a training dataset, it is only slightly better than the MIN, SS and MAX rules. In the following experiments, we only report the results by using the ABF rule.

\subsection{Experiment settings and parameter selection}

We compare the proposed reconstruction based FKP verification method with ABF, denoted by "R-ABF", with state-of-the-art FKP verification methods, including the improved CompCode (ImCompCode) [13], BLPOC [14], LGIC [15] and the local feature integration (LFI) method [16]. Considering that the LGIC scheme is a combination of the CompCode (which employs the image local orientation features) and the BLPOC (which employs the image global Fourier transform features) methods, for fair comparison we will first compare R-ABF with ImCompCode, BLPOC and LFI, and then couple R-ABF with BLPOC and compare it with the LGIC method.

In the proposed method, the query image reconstruction can be regularized by either $l_{1}$-norm or $l_{2}$-norm, and can be done either globally or patch-by-patch. Therefore, there are four variants of the proposed R-ABF scheme, denoted by R-ABF-g- $l_{1}$, R-ABF-g- $l_{2}$, R-ABF-p- $l_{1}$ and R-ABF-p- $l_{2}$, respectively, where "g" is for "global" and "p" is for "patch". In order to evaluate the proposed R-ABF method more comprehensively, we conduct 3 experiments with different sizes of the gallery set (165, 330 and 660 fingers, respectively). In all the experiments, the gallery set is extracted from the FKP images collected in the first session, while all the FKP samples collected in the second session are used as the probe set (all the 660 fingers).

There are some parameters to set in our algorithm. For the parameters in CompCode, we adopt the settings in the original paper [12]. The uncertain interval is set by letting $t_{1}=0.35$ and $t_{2}=0.39$. The thresholds $t_{1}$ and $t_{2}$ can also be automatically determined based on the training set. By using the "leave-one-out" strategy, each image of one subject is matched with all the other images in the training set to obtain the distribution of matching distances. The point where the false acceptance rate equals to the false rejection rate is taken as the decision threshold $t$, and the width of the uncertain interval (refer to Fig. 3) can be set as $w_{0}$. Then $t_{1}$ and $t_{2}$ can be determined as: $t_{1}=t-w_{0} / 2$ and $t_{2}=t+w_{0} / 2$. By using this strategy, the computed $t_{1}$ and $t_{2}$ are 0.34 and 0.38 , respectively, which are very close to the manually set thresholds (i.e., 0.35 and 0.39 ).

In the global reconstruction scheme, we set the parameter $\lambda$ in dictionary learning (i.e., in Eq. (5)) as 0.1 by experience. The selection of parameter $\lambda$ in dictionary learning has small influence on the final verification result in terms of EER. The parameter $\lambda$ for $l_{1}$-regularized coding in Eq. (6) and $l_{2}$-regularized 
coding in Eq. (8) is related to the number of atoms in dictionary $\boldsymbol{D}$. We determine it by the following criterion: $\lambda=0.5 \times(n / 990)^{2}$ in $l_{1}$-regularized coding and $\lambda=(n / 990)^{2}$ in $l_{2}$-regularized coding, where $n$ is the number of atoms in the dictionary. In patch based reconstruction, the parameter $\lambda$ in dictionary learning and coding is set as $1 / 10$ of that in global reconstruction. The partition of patches is discussed in Section 6.5.

Our method needs to train a dictionary $\boldsymbol{D}$ with atom number $p \leq k$, where $k$ is the number of samples in gallery set $\boldsymbol{X}$. If $p$ is too small, much information contained in the gallery sample set $\boldsymbol{X}$ can be lost and thus the test sample may not be well represented by the learned dictionary $\boldsymbol{D}$. On the other hand, the commonly used $l_{1}$-minmization solvers such as $l_{1} l s$ have an empirical complexity of $O\left(z^{2} p^{1.3}\right)[18](z$ is the dimension of samples). So if $p$ is big, the computational cost can be high. To balance the computational cost and the representation capability, we set the ratio of $p$ to $k$ as 0.7 in our method, and this configuration leads to satisfying experimental results.

\subsection{FKP verification results}

\subsubsection{Experiment 1}

In this experiment, only the FKP images of the first 165 (out of the 660 ) fingers (i.e., $165 \times 6=990$ samples) are used as the gallery set. Hence, there are 5,940 genuine matchings and 3,914,460 imposter matchings, respectively. Fig. 9(a) plots the DET (Detection Error Tradeoff) curves, which are the plots of false rejection rates (FRR) against false acceptance rates (FAR) for all possible thresholds. Table 4 lists the results of competing methods in terms of EER and decidability index.

It can be seen that the R-ABF methods outperform much ImCompCode, BLPOC and LFI. This validates that the reconstruction of query sample $y$ can reduce much the image deformation induced intra-class distance, and the $\mathrm{ABF}$ rule can prevent the less discriminative reconstruction of $\boldsymbol{y}$ from being adopted for final decision making. Among the four variants of R-ABF, the $l_{1}$-regularized ones have higher accuracy than the $l_{2}$-regularized ones, while the patch based ones have higher accuracy than the global based ones. Specifically, R-ABF-p- $l_{1}$ achieves the lowest EER. This is consistent with our discussions in Section 3.

Table 5 lists the percentage of matchings in which the score from $y$ or the reconstruction $\hat{y}$ is selected as the final matching score. We can see that for genuine matchings, about $40 \% \sim 45 \%$ of the matching scores from $\hat{y}$ are selected by the ABF rule. For imposter matchings, about $28 \% \sim 35 \%$ of the matching scores 
from $\hat{y}$ are adopted.

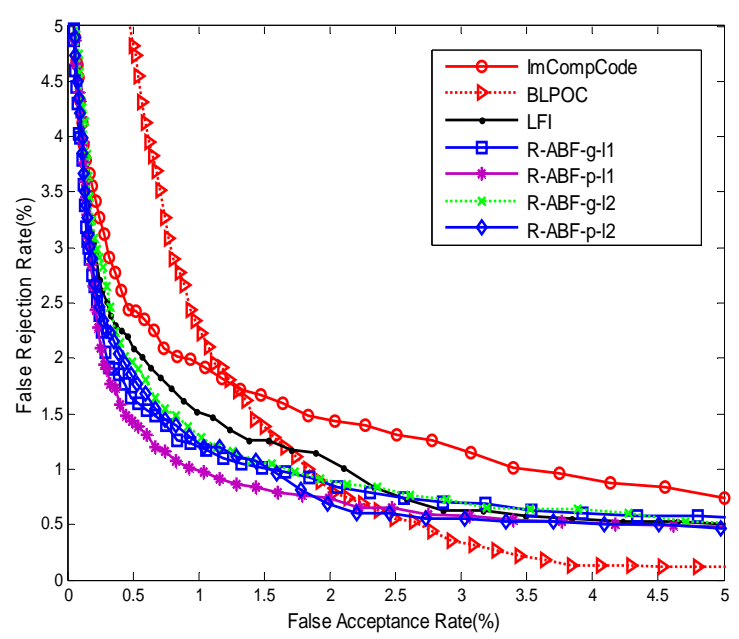

(a)

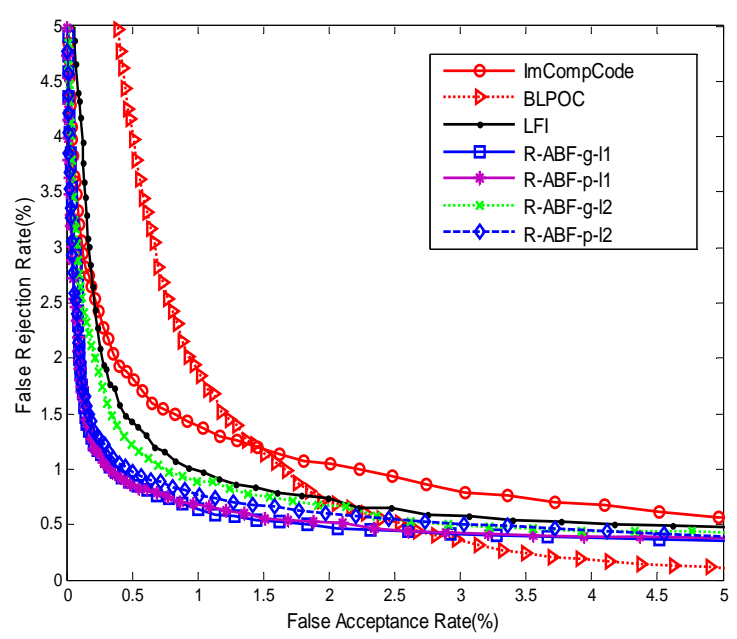

(b)

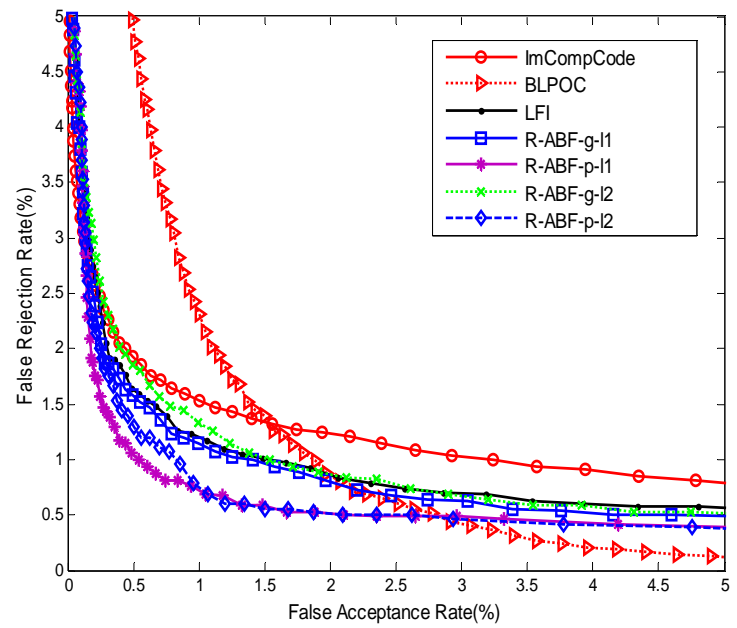

(c)

Figure 9: DET curves by different methods in (a) experiment 1; (b) experiment 2; and (c) experiment 3.

Table 4: EER (\%) values and decidability indices by the competing methods.

\begin{tabular}{||c||c|c||c|c||c|c||}
\hline \multirow{2}{*}{ Method } & \multicolumn{2}{c||}{ Experiment 1 } & \multicolumn{2}{c||}{ Experiment 2 } & \multicolumn{2}{c||}{ Experiment 3 } \\
\cline { 2 - 7 } & $\begin{array}{c}\text { EER } \\
(\%)\end{array}$ & $\begin{array}{c}\text { Decidability } \\
\text { index }\end{array}$ & $\begin{array}{c}\text { EER } \\
(\%)\end{array}$ & $\begin{array}{c}\text { Decidability } \\
\text { index }\end{array}$ & $\begin{array}{c}\text { EER } \\
(\%)\end{array}$ & $\begin{array}{c}\text { Decidability } \\
\text { index }\end{array}$ \\
\hline \hline ImCompCode [17] & 1.62 & 4.1478 & 1.28 & 4.4518 & 1.39 & 4.4302 \\
\hline BLPOC [18] & 1.44 & 4.1925 & 1.35 & 3.4311 & 1.44 & 3.4218 \\
\hline LFI [20] & 1.32 & 4.1951 & 1.03 & 4.4821 & 1.13 & 4.4625 \\
\hline R-ABF-g- $\boldsymbol{I}_{\mathbf{1}}$ & 1.24 & 4.2291 & 0.84 & 4.5699 & 0.94 & $\mathbf{4 . 5 3 0 5}$ \\
\hline R-ABF-p- $\boldsymbol{I}_{\mathbf{1}}$ & $\mathbf{1 . 1 2}$ & $\mathbf{4 . 2 3 0 9}$ & $\mathbf{0 . 7 6}$ & $\mathbf{4 . 6 3 8 5}$ & $\mathbf{0 . 8 2}$ & 4.2572 \\
\hline R-ABF-g- $\boldsymbol{I}_{\mathbf{2}}$ & 1.27 & 4.1948 & 0.91 & 4.5014 & 1.19 & 4.1882 \\
\hline R-ABF-p- $\boldsymbol{I}_{\mathbf{2}}$ & 1.28 & 4.2170 & 0.82 & 4.6188 & 0.92 & 4.2461 \\
\hline
\end{tabular}


Table 5: The percentage (\%) of matchings in which the score from $y$ or $\hat{y}$ is selected.

\begin{tabular}{||c||c|c|c|c||c|c||}
\hline \multirow{2}{*}{ Matching type } & \multicolumn{2}{|c|}{ Experiment 1 } & \multicolumn{2}{c||}{ Experiment 2 } & \multicolumn{2}{c||}{ Experiment 3 } \\
\cline { 2 - 7 } & $\omega_{1}=1$ & $\omega_{2}=1$ & $\omega_{1}=1$ & $\omega_{2}=1$ & $\omega_{1}=1$ & $\omega_{2}=1$ \\
\hline \hline Genuine & 60 & 40 & 55 & 45 & 57 & 43 \\
\hline Imposter & 71.59 & 28.41 & 65.36 & 34.61 & 68.74 & 31.26 \\
\hline
\end{tabular}

\subsubsection{Experiment 2}

In the $2^{\text {nd }}$ experiment, 330 classes are involved in the gallery set. Therefore, the numbers of genuine and imposter matchings are 11,880 and 7,828,920, respectively. Fig. 9 (b) shows the DET curves by the different verification schemes, while the EER values and decidability indices are listed in Table 4. The percentage of matchings in which the score from the reconstruction $\hat{\boldsymbol{y}}$ is selected is listed in Table 5. Again, the proposed R-ABF methods get much better results than ImCompCode, BLPOC and LFI.

\subsubsection{Experiment 3}

At last, all the classes (i.e., all fingers) are involved in the gallery set, and the numbers of genuine and imposter matchings are 23,760 and $15,657,840$, respectively. The DET curves by different verification schemes are illustrated in Fig. 9(c). Table 4 lists the EER values and decidability indices, and Table 5 lists the percentage of matchings in which the score from the reconstruction $\hat{\boldsymbol{y}}$ is selected. Similar conclusions to the previous two experiments can be made.

It can be seen that the EER decreases from Experiment 1 to Experiment 2, and increases from Experiment 2 to Experiment 3. The reason can be as follows. From Experiment 1 to Experiment 3, the number of gallery classes is increasing. The increased number of gallery samples makes the dictionary $\boldsymbol{D}$ more capable to reconstruct the query sample, but it also makes the verification tasks more challenging. There are only 165 gallery classes in Experiment 1, so the learned dictionary $\boldsymbol{D}$ may not be representative enough to reconstruct the query sample. The number of gallery classes is increased to 330 in Experiment 2, and the representativeness of $\boldsymbol{D}$ is much improved so that the FAR and FRR are decreased simultaneously. As a consequence, the overall EER in Experiment 2 is reduced. With 330 gallery classes, the representativeness of learned dictionary $\boldsymbol{D}$ is already good. Thus, the benefit of using 660 gallery classes in Experiment 3 is not big in term of learning dictionary $\boldsymbol{D}$; however, the FAR and FRR are increased simultaneously due to the 
increased number of gallery classes, resulting in a bigger EER than Experiment 2.

\subsection{Integrating with global features}

The LGIC scheme [15] combines CompCode, which employs the image local orientation features, and BLPOC, which employs the global Fourier transform features. In this section, for fair comparison, we also combine R-ABF, which basically employs the image local orientation features, with the BLPOC method in the same way as that in [15]. According to the reconstruction strategy, we denote the fused methods as R-ABF-g- $l_{1}+B L P O C, R-A B F-g-l_{2}+B L P O C, R-A B F-p-l_{1}+B L P O C$ and R-ABF-p- $l_{2}+B L P O C$, respectively. We compare the performance of LGIC with the proposed methods under the same experiment settings as described in Section 6.3. The combination of LFI and BLPOC is also used for more comprehensive comparison. The DET curves of the competing methods are shown in Fig. 10, and the EER values and decidability indices are summarized in Table 6.

From the experimental results in both Sections 6.3 and 6.4, we can see that the proposed R-ABF scheme leads to state-of-the-art verification accuracy, no matter using only the local orientation feature or using both local and global features. Specifically, the R-ABF-p- $l_{1}$ and R-ABF-p- $l_{1}+$ BLPOC methods achieve the best accuracy, respectively. By relaxing the $l_{1}$-regularized sparsity constraint in the reconstruction, the $l_{2}$-regularized reconstruction can also lead to very competitive verification results but with much less complexity, which is a good solution in practical FKP recognition systems. 


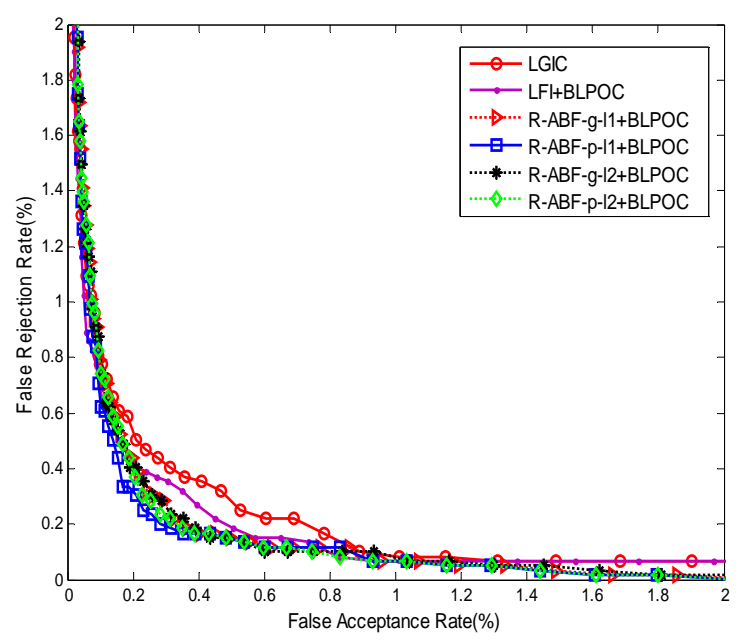

(a)

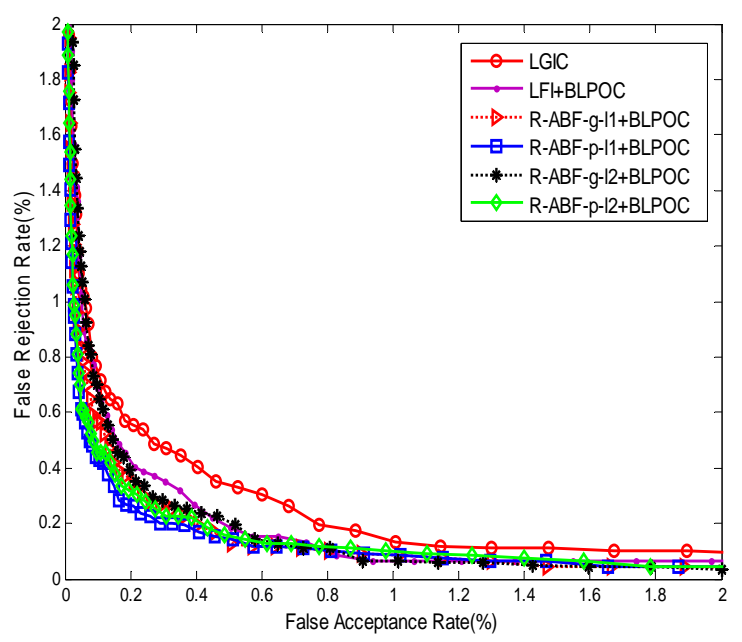

(b)

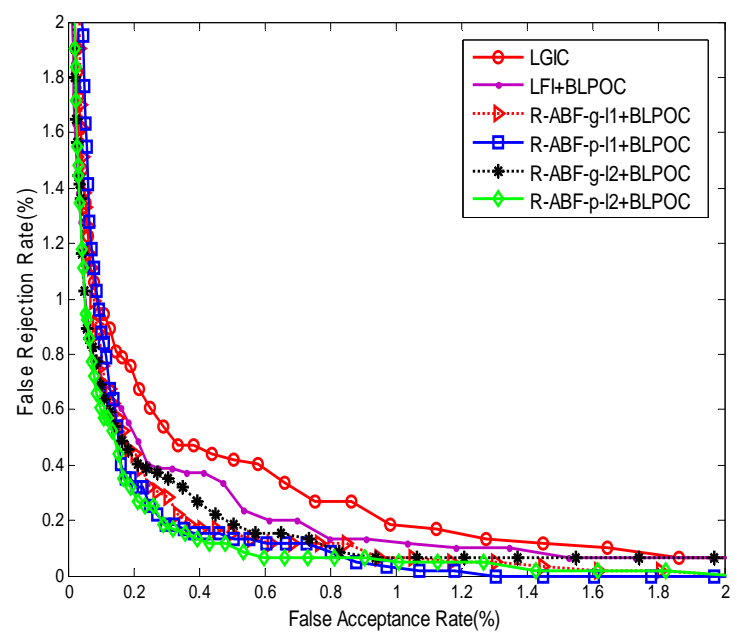

(c)

Figure 10: DET curves by the fused methods in (a) experiment 1; (b) experiment 2; and (c) experiment 3 .

Table 6: EER (\%) values and decidability indices by the competing methods.

\begin{tabular}{|c||c|c||c|c||c|c||}
\hline \multirow{2}{*}{ Method } & \multicolumn{2}{|c||}{ Experiment 1 } & \multicolumn{2}{c||}{ Experiment 2 } & \multicolumn{2}{c||}{ Experiment 3 } \\
\cline { 2 - 7 } & $\begin{array}{c}\text { EER } \\
(\%)\end{array}$ & $\begin{array}{c}\text { Decidability } \\
\text { index }\end{array}$ & $\begin{array}{c}\text { EER } \\
(\%)\end{array}$ & $\begin{array}{c}\text { Decidability } \\
\text { index }\end{array}$ & $\begin{array}{c}\text { EER } \\
(\%)\end{array}$ & $\begin{array}{c}\text { Decidability } \\
\text { index }\end{array}$ \\
\hline \hline LGIC [19] & 0.38 & 4.3882 & 0.39 & 4.6182 & 0.41 & 4.6522 \\
\hline LFI+BLPOC & 0.33 & 4.3946 & 0.35 & 4.6378 & 0.35 & 4.6635 \\
\hline R-ABF-g- $\boldsymbol{I}_{\mathbf{1}}+$ BLPOC & 0.29 & $\mathbf{4 . 4 1 9 7}$ & 0.25 & 4.6886 & 0.29 & $\mathbf{4 . 6 9 4 0}$ \\
\hline R-ABF-- $\boldsymbol{I}_{\mathbf{1}}+$ BLPOC & $\mathbf{0 . 2 5}$ & 4.4150 & $\mathbf{0 . 2 1}$ & $\mathbf{4 . 7 4 7 9}$ & $\mathbf{0 . 2 2}$ & 4.4823 \\
\hline R-ABF-g- $\boldsymbol{I}_{\mathbf{2}}+$ BLPOC & 0.29 & 4.4048 & 0.29 & 4.5937 & 0.33 & 4.4641 \\
\hline R-ABF-p- $\boldsymbol{I}_{\mathbf{2}}+\mathbf{B L P O C}$ & 0.27 & 4.4076 & 0.26 & 4.7354 & 0.26 & 4.4764 \\
\hline
\end{tabular}




\subsection{Discussions}

In the proposed method, we learn a dictionary $\boldsymbol{D}$ from the gallery set to reconstruct a query sample. When a new subject is enrolled, we can update $\boldsymbol{D}$ by solving Eq. (5). However, if the dataset has a large scale, this can be very costly. Fortunately, it is not necessary to update $\boldsymbol{D}$ for a new enrollment in large-scale dataset.

The dictionary $\boldsymbol{D}$ in our algorithm is just used for reconstruction, and it is not used in the classification stage. This is very different from the works in [21-23], where the atoms in the dictionary have class labels and they will be used to calculate the class-specific distances for classification. The role of dictionary $\boldsymbol{D}$ in our work is similar to the dictionaries in image restoration such as K-SVD [19]. There are no class labels of the atoms in $\boldsymbol{D}$, and $\boldsymbol{D}$ is a universal dictionary shared by all classes. Once enough gallery classes are involved in learning the dictionary $\boldsymbol{D}$, this $\boldsymbol{D}$ will be able to well represent any FKP image. Therefore, when there are some new enrollments, we actually do not need to update the dictionary. Kindly note that the online learning algorithms in $[33,34]$ aim for updating classifiers when new individuals are enrolled. The problem is very different from ours.

Let us use two experiments to validate the above statement. We use the first 600 classes out of the 660 classes in the PolyU FKP database as the gallery set (6 samples per class in the first session) to learn a dictionary, denoted by $\boldsymbol{D}_{1}$. We then use all the 660 classes to learn another dictionary, denoted by $\boldsymbol{D}_{2}$. In the first experiment, we take $\boldsymbol{D}_{1}$ as the dictionary and take the 60 new classes ( 6 samples per class) as the query set, and the EER is $0.8 \%$ by the proposed R-ABF-g- $l_{2}$ method. If we take $\boldsymbol{D}_{2}$ as the dictionary and take the 60 same classes as the query set, the EER is $0.76 \%$ by R-ABF-g- $l_{2}$. In the second experiment, with $\boldsymbol{D}_{1}$ and using all the 660 classes as the query set (6 samples per class in the second session), the EER is $1.21 \%$ by R-ABF-g- $l_{2}$, while with $\boldsymbol{D}_{2}$ and using all the 660 classes as the query set, the EER is $1.19 \%$ by R-ABF-g- $l_{2}$. Clearly, in both the two experiments, the EER values by $\boldsymbol{D}_{1}$ and $\boldsymbol{D}_{2}$ are very close, implying that there is no necessary to further update the dictionary since $\boldsymbol{D}_{1}$ is already good in reconstruction.

If we do want to update the dictionary $\boldsymbol{D}$ when new enrollments come, there are two strategies to save cost. First, we can update $\boldsymbol{D}$ once a batch of new enrollments is available. Second, we can let the new dictionary be $\boldsymbol{D}_{\text {new }}=\left[\begin{array}{ll}\boldsymbol{D}_{o} & \boldsymbol{D}_{a}\end{array}\right]$, where $\boldsymbol{D}_{o}$ is the old dictionary and $\boldsymbol{D}_{a}$ includes the new atoms to be added. In this way, we only need to learn the several new atoms by using dictionary learning algorithms such as [21]. 


\section{Conclusions}

This paper presented a novel reconstruction based finger-knuckle-print (FKP) verification method to reduce the false rejections caused by finger pose variations in data collection process. For an input query image whose matching distance falls into the uncertain interval, we reconstructed a new version of it by using a dictionary learned from the gallery set. Then a new matching distance can be obtained. An adaptive binary fusion (ABF) rule was then proposed to fuse the two matching distances for the final decision making. The proposed reconstruction based FKP verification with $\mathrm{ABF}$, denoted by $\mathrm{R}-\mathrm{ABF}$, can effectively reduce the false rejections without increasing much the false acceptances. Our extensive experimental results demonstrated that the R-ABF can result in much lower equal error rate than existing state-of-the-art methods.

\section{References}

[1] K. Delac, M. Grgic, "Face Recognition," I-Tech Education and Publishing, 2007.

[2] H. Wechsler, "Reliable Face Recognition Methods-System Design, Implemen-tation and Evaluation," Springer, 2006.

[3] D. Maltoni, D. Maio, A.K. Jain, S. Prabhakar, "Handbook of Fingerprint Recognition," Springer, 2003.

[4] Q. Zhao, D. Zhang, L. Zhang, N. Luo, "Adaptive fingerprint pore modeling and extraction," Pattern Recognition, vol.43, no.8, pp. 2833-2844, Aug. 2010.

[5] J. Daugman, "How iris recognition works," IEEE Transactions on Circuits and Systems for Video Technology, vol. 14, no.1, pp. 21-30, Jan. 2004.

[6] W. Dong, Z. Sun, T. Tan, "Iris matching based on personalized weight map," IEEE Trans. Pattern Analysis and Machine Intelligence, vol. 33, no. 9, pp. 1744-1757, Sep. 2011.

[7] D. Zhang, W. K. Kong, J. You, M. Wong, "Online palmprint identification," IEEE Trans. Pattern Analysis and Machine Intelligence, vol. 25, no. 9, pp. 1041-1050, Sep. 2003.

[8] W. K. Kong, D. Zhang, "Competitive coding scheme for palmprint verification," In Proc. IEEE Int. Conf. Pattern Recognition, Sep. 2004, vol.1, pp. 520-523.

[9] D. Zhang, G. Lu, W. Li, L. Zhang, N. Luo, "Palmprint recognition using 3-D information," IEEE Transactions on Systems, Man, and Cybernetics, Part C: Applications and Reviews, vol. 39, no. 5, pp. 505-519, Sep. 2009.

[10] R. Sanchez-Reillo, C. Sanchez-Avila, A. Gonzalez-Marcos, "Biometric identification through hand geometry measurements," IEEE Trans. Pattern Analysis and Machine Intelligence, vol. 22, no. 10, pp. 1168-1171, Oct. 2000.

[11] J.G. Wang, W.Y. Yau, A. Suwandy, E. Sung, "Personal recognition by fusing palmprint and palm vein images based on "Lapacianpalm" representation," Pattern Recognition, vol. 41, no. 5, pp. 1531-1544, May. 2008.

[12] L. Zhang, L. Zhang, D. Zhang, "Finger-knuckle-print: a new biometric identifier," In Proc. IEEE Int. Conf. Image Process, Nov. 2009, pp.1981-1984.

[13] L. Zhang, L. Zhang, D. Zhang, H. Zhu, "Online finger-knuckle-print verification for personal authentication," Pattern Recognition, vol. 43, no. 7, pp. 2560-2571, Jul. 2010.

[14] L. Zhang, L. Zhang, D. Zhang, "Finger-knuckle-print verification based on band-limited phase-only correlation," In Proc. International Conference on Computer Analysis of Images and Patterns, Sep. 2009, pp. 141-148.

[15] L. Zhang, L. Zhang, D. Zhang, H. Zhu, "Ensemble of local and global information for 
finger-knuckle-print recognition," Pattern Recognition, vol. 44, no. 9, pp. 1990-1998, Sep. 2011.

[16] L. Zhang, L. Zhang, D. Zhang, Z. Guo, "Phase congruency induced local features for finger-knuckle-print recognition," Pattern Recognition, vol. 45, no. 7, pp. 2522-2531, Jul. 2012.

[17] E. Candès and J. Romberg, " $L_{1}$-magic: Recovery of sparse signals via convex programming," http://www.acm.cal tech.edu/l1magic/, 2005.

[18] S.J. Kim, K. Koh, M. Lustig, S. Boyd, and D. Gorinevsky, "An interior-point method for large-scale $l_{1}$-regularized least squares," IEEE Journal on Selected Topics in Signal Processing, vol. 1,no. 4, pp. 606-617, Dec. 2007.

[19] M. Aharon, M. Elad, and A. Bruckstein, "K-SVD: An algorithm for designing overcomplete dictionaries for sparse representation," IEEE Transactions on Signal Processing, vol. 54, no. 11, pp. 4311-4322, Nov. 2006.

[20] R.Rubinstein, A.M. Bruckstein, and M. Elad, "Dictionaries for sparse representation modeling," Proceedings of IEEE, vol. 98, no. 6, pp. 1045-1057, Jun. 2010.

[21] M. Yang, L. Zhang, J. Yang, and D. Zhang, "Metaface learning for sparse representation based face recognition," In Proc. IEEE Int. Conf. Image Process, Sep. 2010, pp.1601-1604.

[22] J. Wright, A. Yang, A. Ganesh, S. Sastry, Y. Ma, "Robust face recognition via sparse representation," IEEE Trans. Pattern Anal. Mach. Intell., vol. 31, no. 2, pp. 210-227, Feb. 2009.

[23] M. Yang, L. Zhang, X. Feng and D. Zhang, "Fisher Discrimination Dictionary Learning for Sparse Representation," Proc. IEEE Int. Conf. Computer Vision, Nov. 2011, pp. 543-550.

[24] S. Wang, L. Zhang, Y. Liang and Q. Pan, "Semi-Coupled Dictionary Learning with Applications to Image Super-Resolution and Photo-Sketch Image Synthesis," Proc. IEEE Conf. Computer Vision and Pattern Recognition, June. 2012, pp. 2216-2223.

[25] A. Beck and M. Teboulle, "A fast iterative shrinkage-thresholding algorithm for linear inverse problems," SIAM. J. Imaging Science, vol. 2, no. 1, pp. 183-202, Jan. 2009.

[26] J. Yang and Y. Zhang, "Alternating direction algorithms for $l_{1}$-problems in compressive sensing," arXiv:0912.1185, 2009.

[27] D. Malioutove, M. Cetin, and A. Willsky, "Homotopy continuation for sparse signal representation," Proc. IEEE Int'l Conf. Acoustics, Speech and Signal Processing, Mar. 2005, vol.5, pp. 733-736.

[28] R. Snelick, U. Uludag, A. Mink, M. Indovina, and A. Jain, "Large-scale evaluation of multimodal biometric authentication using state-of-the-art systems," IEEE Trans. Pattern Analysis and Machine Intelligence, vol. 27, no. 3, pp. 450-455, Mar. 2005.

[29] A. Kumar, V. Kanhangad, D. Zhang, "A New Framework for Adaptive Multimodal Biometrics Management," IEEE Trans. Information Forensics and Security, vol. 5, no. 1, pp. 92-102, Mar. 2010.

[30] R. C. Eberhart and J. Kennedy, "Swarm Intelligence," San Diego, CA: Morgan Kaufmann, 2001.

[31] J. Daugman, "The importance of being random: statistical principles of iris recognition," Pattern Recognition, vol. 36, no. 2, pp. 279-291, Feb. 2003.

[32] PolyU Palmprint Database 2006 [Online]. Available: http://www.comp.polyu.edu.hk/ biometrics.

[33] D. Masip, A. Lapedriza, J. Vitria, "Boosted Online Learning for Face Recogition," IEEE Transactions on Systems, Man, and Cybernetics, Part B: Cybernetics, vol. 39, no. 2, pp. 530-538, Apr. 2009.

[34] R. Singh, M. Vatsa, A. Ross, A. Noore, " Biometric Classifier Update using OnlineLearning: A Case Study in Near Infrared Face Verification," Image and Vision Computing, vol. 28, no. 7, pp. 1098-1105, Jul. 2010. 